\title{
Acute aortic dissection with coronary ostium involvement and aortic valve regurgitation: Three- dimensional visualization with multislice computed tomography
}

Gudrun Maria Feuchtner, MD, ${ }^{\text {a }}$ Thomas Schachner, MD, ${ }^{\mathrm{c}}$ Guy Friedrich, MD, ${ }^{\mathrm{b}}$ Herwig Antretter, MD, ${ }^{\mathrm{c}}$ Johannes Bonatti, MD, ${ }^{\mathrm{c}}$ and Dieter zur Nedden, MD, ${ }^{\mathrm{a}}$ Innsbruck, Austria

Extra material is available online. $\mathcal{\beta}$
From the Departments of Radiology II, ${ }^{\mathrm{a}}$ Cardiology, ${ }^{\mathrm{b}}$ and Cardiac Surgery, Medical University Innsbruck, Innsbruck, Austria.

Received for publication March 1, 2005; accepted for publication March 23, 2005.

Address for reprints: Gudrun Maria Feuchtner, MD, Department of Radiology II, Medical University Innsbruck, Anichstrasse 35, A-6020 Innsbruck, Austria (E-mail: Gudrun.Feuchtner@uibk.ac.at).

J Thorac Cardiovasc Surg 2005;130:587-8

$0022-5223 / \$ 30.00$

Copyright $\odot 2005$ by The American Association for Thoracic Surgery doi:10.1016/j.jtcvs.2005.03.030
A

67-year-old man was transferred to the emergency department with

severe chest pain and signs of cardiac decompensation. The electrocardiogram (ECG) showed marked ST-segment depression in leads II, III, aVF, and V2-V6, suggesting acute anterior and true posterior myocardial ischemia. Cardiac enzymes and troponin $\mathrm{T}$ levels were normal on admission. The patient had a history of endovascular stent graft placement in the ascending aorta and the aortic arch 3 years ago.

Multislice computed tomographic (MSCT) angiography of the thoracic aorta and coronary arteries with a latest-generation 16row MSCT scanner (Sensation 16, Siemens Medical Solutions) was performed immediately (collimation, $16 \times 0.75 \mathrm{~mm}$; gantry rotation time, 0.42 second).

MSCT demonstrated an acute type A aortic dissection, with an intimal flap originating from the proximal anastomosis of the stent graft located at the aortic arch (Figure 1). The dissection flap involved the right and left coronary ostium (Figure 2) and approached the aortic valve (Figure E1). By using retrospective ECG gating, images were reconstructed within diastole, at which aortic valve leaflets did not close up, suggesting aortic regurgitation (Figure E1).

Emergency surgical repair of the ascending aorta with a $30-\mathrm{mm}$ prosthesis was performed. Intraoperative echocardiography confirmed severe aortic regurgitation classified as grade IV. The patient presented with myocardial infarction (serum creatine kinase, $2.271 \mathrm{U} / \mathrm{L}$; creatine kinase $\mathrm{MB}, 263 \mathrm{U} / \mathrm{L}$; relative $11.6 \%$, and troponin $\mathrm{T}, 517.5 \mathrm{mg} / \mathrm{dL}) 7$ hours after admission. The intensive care unit stay of 35 days was complicated by supraventricular tachycardia and renal failure requiring hemofiltration. The patient was discharged on the 56th hospital day.

In summary, by using a recently introduced, latest-generation, 16-channel MSCT scanner, diagnosis of life-threatening acute aortic dissection complicated by myocardial infarction and cardiac decompensation was given quickly and accurately within a few minutes without requiring any further invasive diagnostic testing. Successful emergency surgical repair of the ascending aorta could be performed immediately on the basis of exclusively MSCT findings.

Currently available advanced MSCT technology offers high spatial resolution $\left(0.5 \times 0.5 \times 0.6 \mathrm{~mm}^{3}\right)$ and low temporal resolution $(>105 \mathrm{~ms})$ imaging, thus permitting a display of coronary ostium involvement, which led to myocardial ischemia in our 


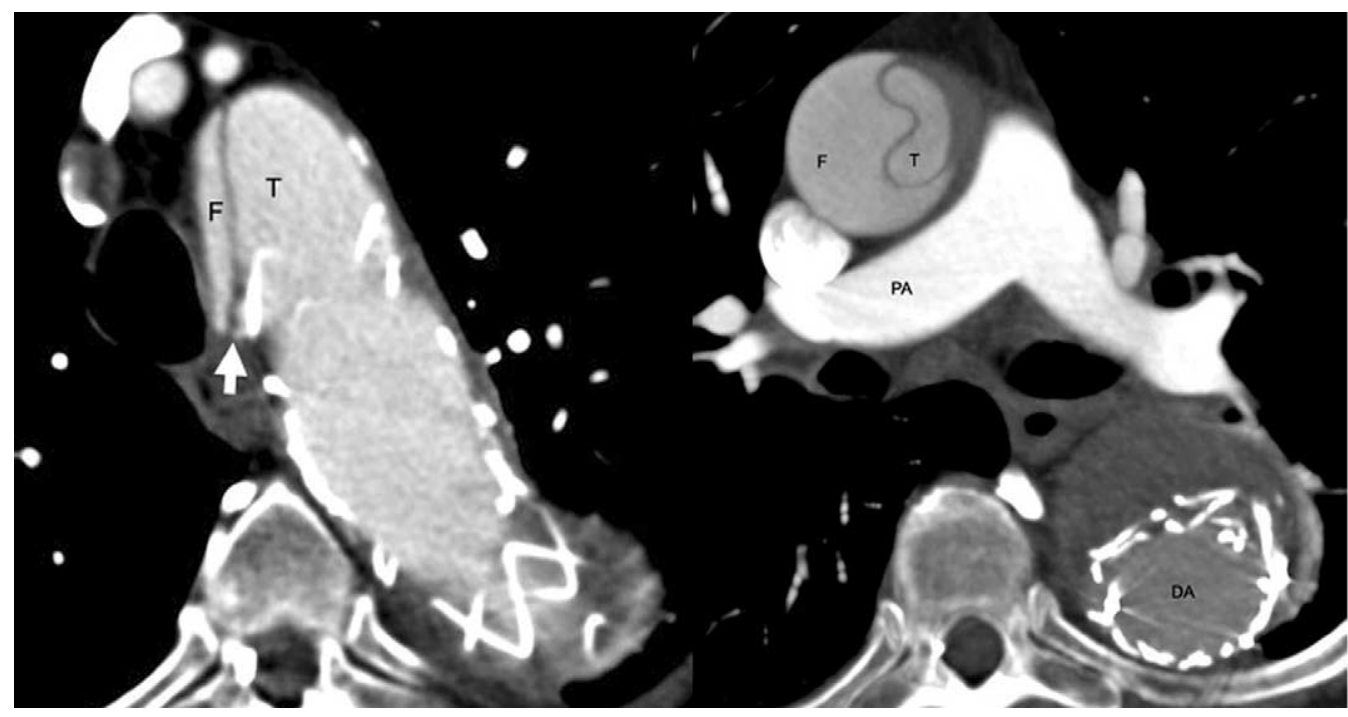

Figure 1. Acute ascending aortic dissection. The site of the intimal tear was the stent graft anastomosis (white arrow). The intimal flap forms a true (T) and false (F) lumen. Note the partially thrombosed stent graft, which is placed in the descending aorta (DA). Images are displayed with multiplanar reformation. PA, Pulmonary artery.

case. In addition, by applying retrospective ECG gating, visualization of severe aortic valve regurgitation was feasible. Subsequently, MSCT might provide interesting information regarding preoperative planning before ascending aortic surgical intervention.

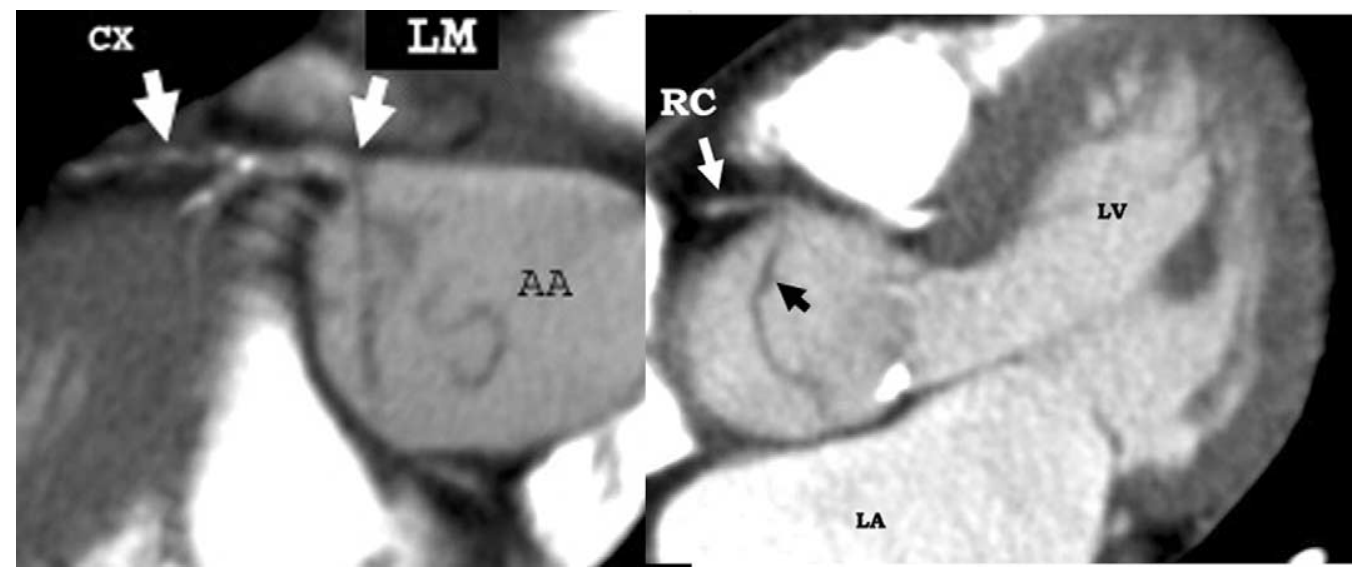

Figure 2. The dissection involved the left and right coronary ostium, which caused acute myocardial ischemia. $L A$, Left atrium; $C X$, circumflex artery; $R C$, right coronary artery; $L M$, left main coronary artery; $A A$, ascending aorta; $L V$, left ventricle. 


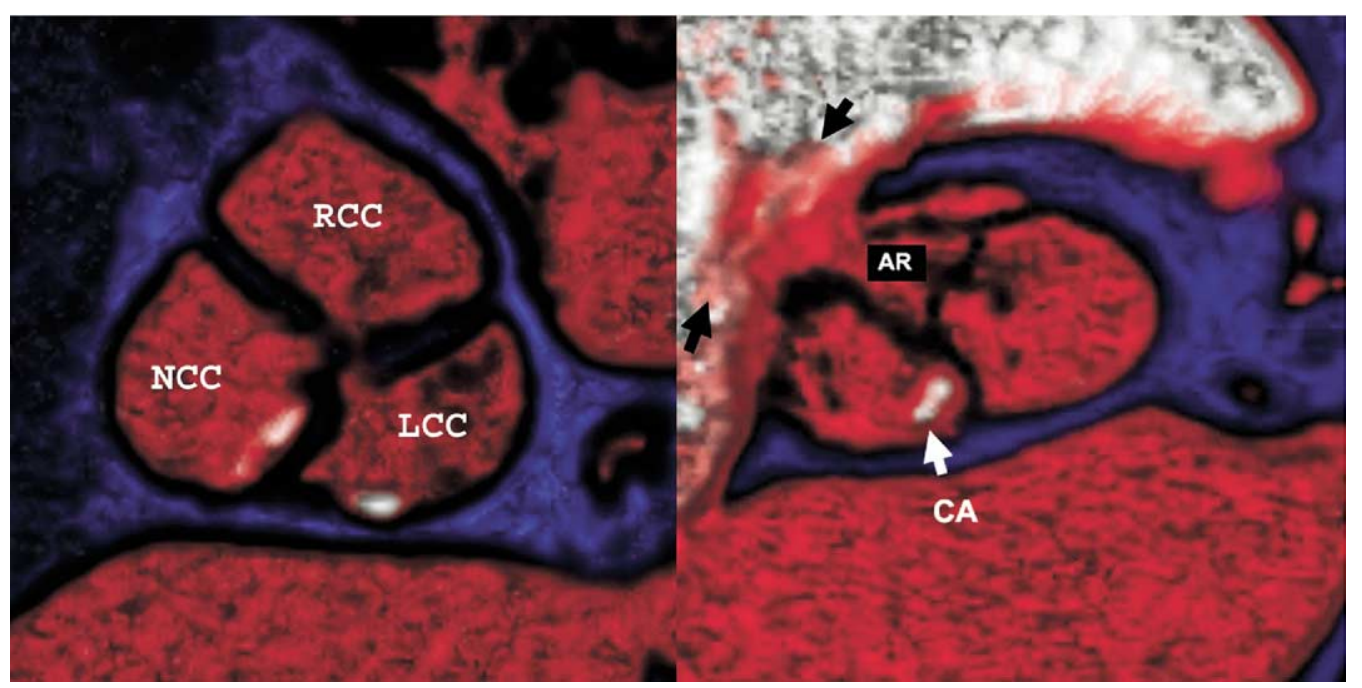

Figure E1. Left, The aortic valve is displayed closed without aortic regurgitation (normal finding). RCC, Right coronary cusp; $L C C$, left coronary cusp; NCC, noncoronary cusp. Right, Three-dimensional MSCT image of the aortic valve before surgical intervention is displayed. Aortic valve leaflets did not close up during diastole, and a central triangular leakage could be displayed suggesting aortic valve regurgitation (AR). Black arrows denote the regurgitation jet, which is displayed 3-D by applying volume-rendering technique. $C A$, Aortic valve calcification. 